\title{
Fully Endoscopic Resection of Intra-Axial Brain Lesions Using Neuronavigated Pediatric Anoscope
}

\author{
Murat KUTLAY, Cahit KURAL, Ilker SOLMAZ, Ozkan TEHLI, Caglar TEMIZ, Mehmet DANEYEMEZ, Yusuf IZCI \\ Gulhane Military Medical Academy, Department of Neurosurgery, Ankara, Turkey
}

\section{ABSTRACT}

AIM: To present a new simple disposable tubular retractor which provides fully endoscopic resections of the intraaxial brain lesions. MATERIAL and METHODS: A total of 13 patients underwent surgical resection of an intraaxial brain lesion larger than $3 \mathrm{~cm}$ with a fully endoscopic approach using the navigation-guided pediatric anoscope. The anoscope was adapted to serve as a tubular retractor. All lesions were resected under endoscopic visualization and navigation guidance. There were 7 men and 6 women with a mean age of 49.6 years (range, 19-76 years). Lesion location was as follows: frontal $(n=4)$, parietal $(n=1)$, frontoparietal $(n=2)$, temporal $(n=2)$, and intraventricular $(n=4)$.

RESULTS: With the use of this technique, preoperative goals of surgery were met in all patients. Gross total resection of the lesions was achieved in 7 of 13 patients (53.8\%), near-total resection in 3 patients $(23.1 \%)$ and subtotal resection in $3(23.1 \%)$ patients. The histological diagnosis included 2 metastases, 5 (38.5\%) glioblastomas, 3 meningiomas, 2 low grade gliomas and one oligodendroglioma. There were no complications related to the surgical procedure. Duration of surgery ranged from 60 to 110 minutes, with an average 90 minutes. The average postoperative hospital stay was 2.7 days.

CONCLUSION: Endoscopic resection of deep-seated brain lesions with the neuronavigated tubular retractor is a safe and an effective technique and may be a feasible alternative to conventional microsurgical or endoscope-assisted methods in selected patients. The modified transparent plastic pediatric anoscope can be used as a tubular retractor and it is easy to apply, simple, lightweight, inexpensive and effective.

KEYWORDS: Endoscopy, Navigation, Brain tumor, Tubular retractor

\section{INTRODUCTION}

Surgical access to deep-seated intraparenchymal and intraventricular brain lesions without damage to the surrounding brain tissue is difficult because corticotomy and brain retraction are usually required to reach these lesions $(2,10,15,20$, $27,28)$. In the past and currently, the most common instruments used in brain retraction procedures are various types of flat-spatula retractors which do not exert equally distributed pressure (10). Therefore, the occurrence of seizures, brain edema, focal neurological deficits and vascular damage after the improper use of these traditional fixed and rigid brain retractors is not uncommon $(12,14,29,30,33,36,37)$. In an ef- fort to facilitate the resection of deep-seated brain lesions with minimal injury to the surrounding neural tissue, different types of tubular retraction systems have been developed. Kelly et al. $(18,19)$ first described the use of a tubular retractor system for the treatment of the deep-seated intracranial tumors. Then, many groups have published their experience. The use of the tubular retractors allowed access to tumors and vascular malformations in a variety of locations $(1,2,5,7-14,16$, 24-28, 33).

On the other hand, minimal invasive neurosurgery is becoming more and more standard in neurosurgical procedures. The application of neurosurgical endoscopy was first described 
by Apuzzo et al. in 1977 (3). Recently, neuroendoscopic procedures have been widely used for the treatment of various brain lesions, and its role in the management of intraventricular, and intracranial extra-axial (e.g. posterior fossa) and as well as skull base tumors is well established (6, $7,9,11,13,15,17,26,35)$. Although the intraparenchymal space not provides natural medium for light dispersion, and the endoscopic view is not sufficient to remove tumors, the introduction of tubular retractor has enabled neurosurgeons to endoscopic approach deep-seated intra-axial lesions. Endoscopic visualization and removal of intra-axial brain tumors using stereotactic guidance was first described in 1980 (32). Over the years, the technique has been improved and several groups have shown that the endoscopic resection of deep brain lesion using tubular retractor may have some advantages over traditional craniotomy $(1,4,10,15,16,20-25$, 34). However, despite the success with the neuronavigationguided endoscopic approach, this technique never gained widespread acceptance.

Currently, different types of tubular retractor systems are available $(8,10,15,24,27,31,33)$ but most of them have their own limitations. These limitations include; their need for frame- based or frameless stereotactic systems (not available in all neurosurgical centers), difficulties in combination with the navigation systems, their bulky and metallic or hard plastic nature (preventing visualization of surrounding tissue), difficulties in their surgical manipulations, and also their fixation problems. Additionally, some of them have their unique setbacks and are very costly. Because of these limitations, widespread adoption of this surgical technique was limited.

In this report, we described a new tubular retractor which is easy to apply. To enable a more efficient use of an endoscope in removing intraparenchymal deep-seated lesions, a transparent pediatric anoscope was used as a tubular retractor. It was combined with frameless image guidance. We reported our institutional experience in 13 cases and discussed the advantages and limitations of this technique.

\section{MATERIAL and METHODS}

\section{Patient Population}

We retrospectively reviewed our case series of intraparenchymal brain lesion treated with fully endoscopic surgery using navigation-guided tubular retractor. In our clinic, because the lesions smaller than $3 \mathrm{~cm}$ in diameter were resected with 'the endoport technique' described previously by Harris et al. (11), and Kassam et al. (16), only the lesions larger than $3 \mathrm{~cm}$ in diameter which were removed with the aid of the disposable pediatric were included. A total of 13 patients ( 7 female, 6 male) with intra-axial lesions were operated using this technique. Patient age ranged from 19 to 76 years (mean 49.6 years). The clinical, radiological and histological features of the patients are summarized in Table I.

Table I: The Demographic, Clinical, Radiological and Surgical Features of the Patients

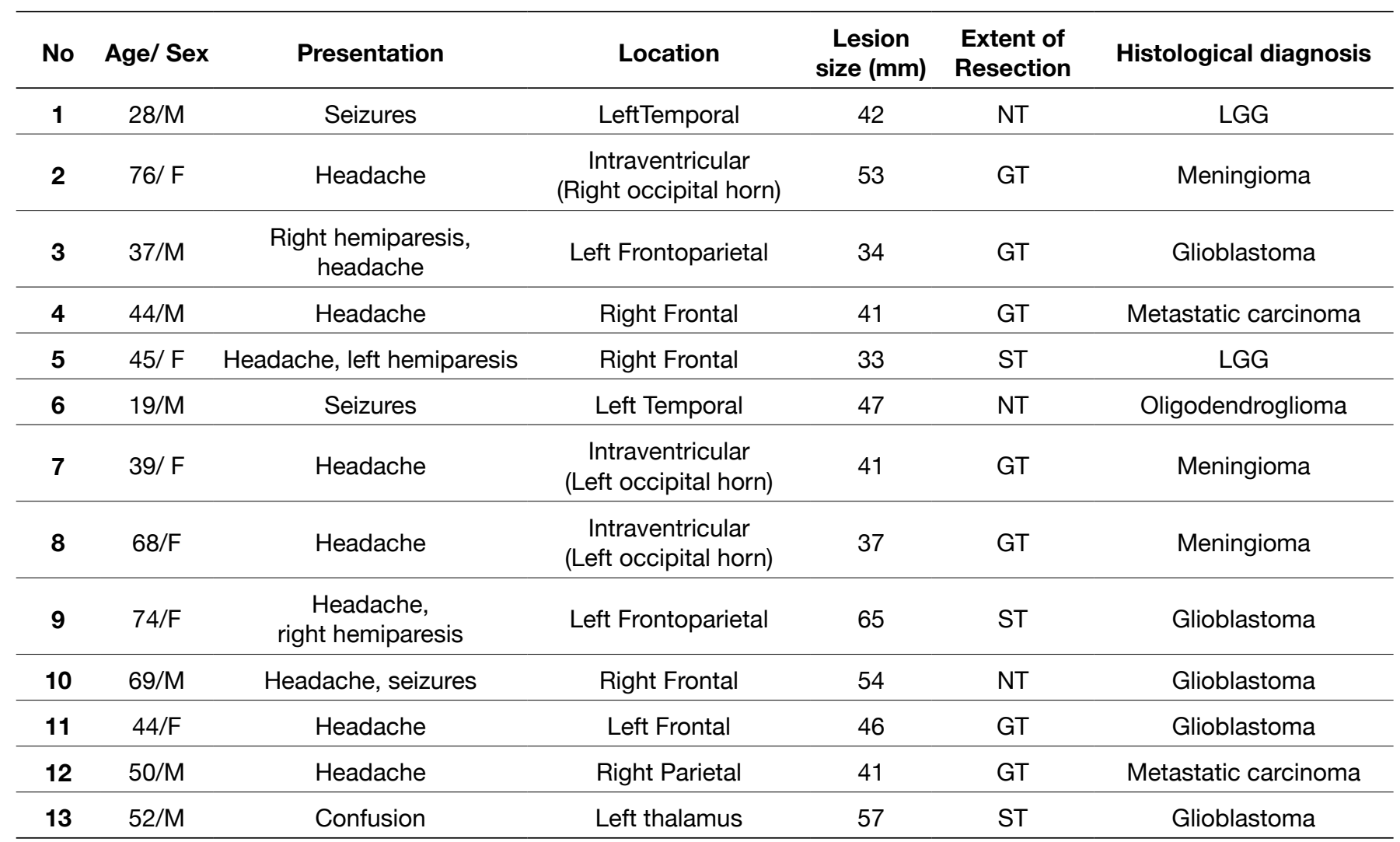

GT: Gross Total resection, NT: Near-Total resection, ST: Subtotal resection, LGG: Low grade glioma. 


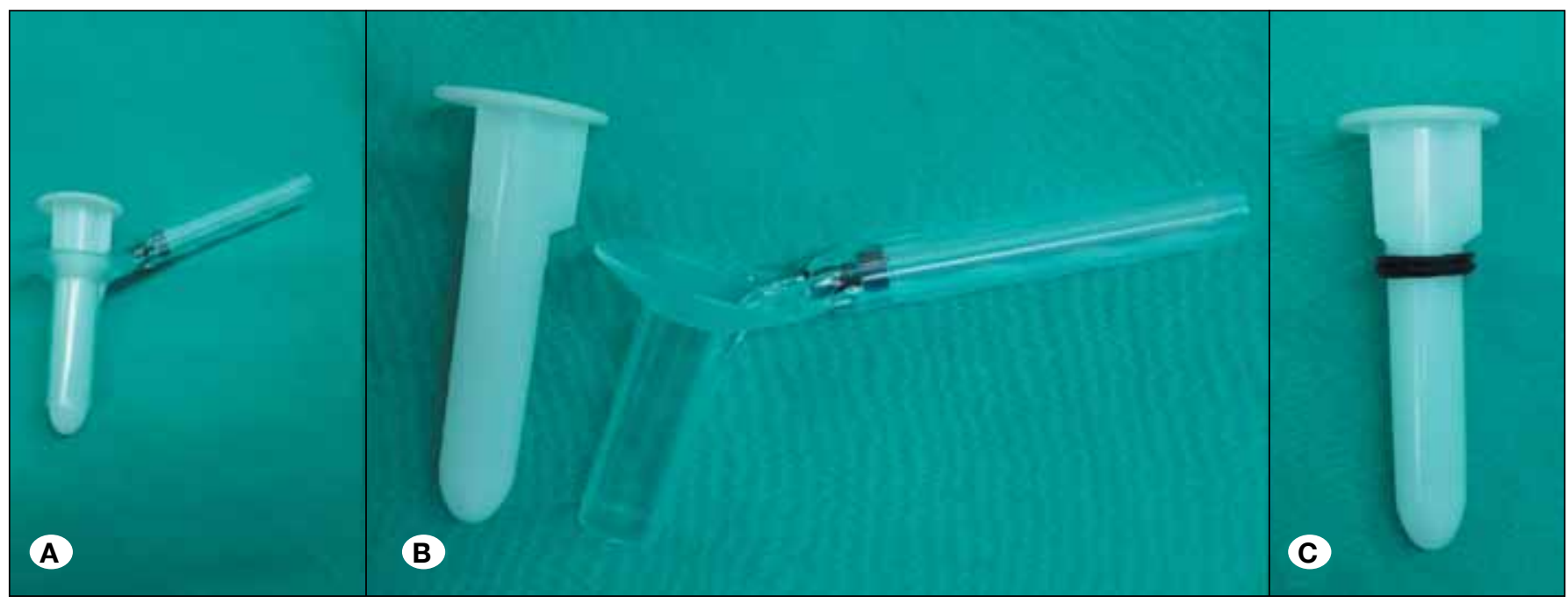

Figure 1: A) Picture of the pediatric anoscope (Ref. A. 4019. Sapimed S.p.A-Alessandria, Italy) used as the tubular retractor. B) It is composed of transparent plastic and it has an inner obturator. C) Photograph showing the obturator. To decrease the distance between the distal tips of the tube and the obturator, a plastic stopper (plastic piston obtained from a 20 ml plastic syringe) was placed at the end of the obturator.

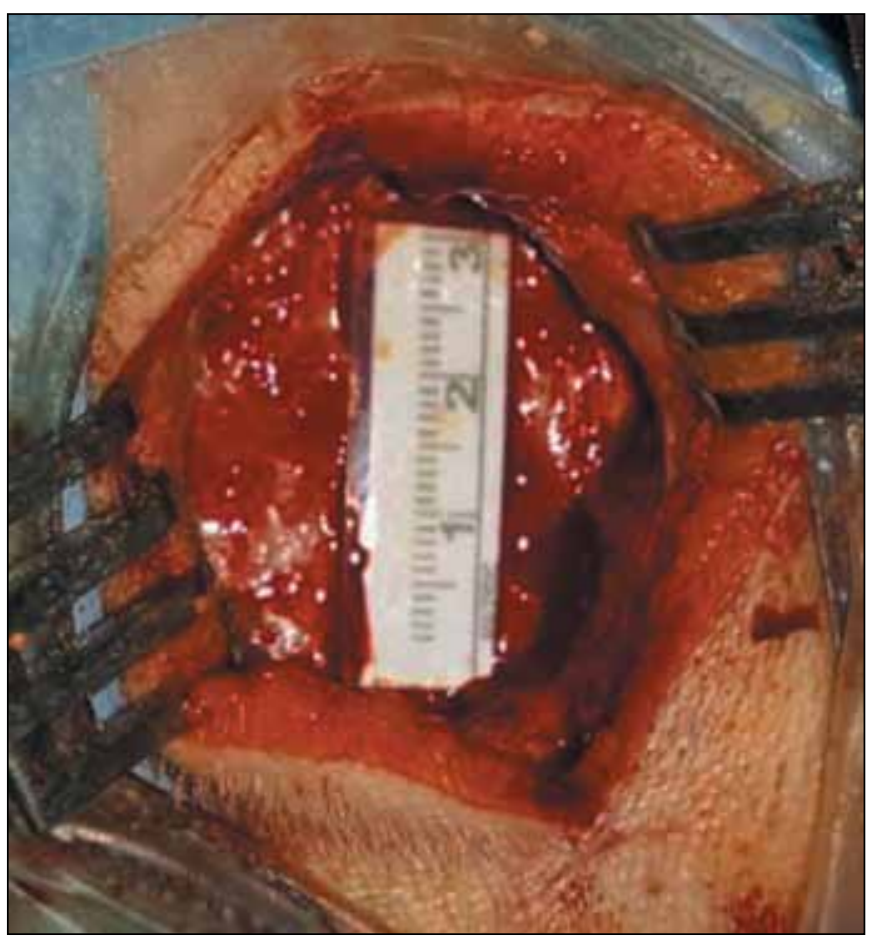

Figure 2: Intraoperative photograph showing craniotomy of approximately $3 \mathrm{~cm}$ in diameter.

There were 10 intraparenchymal lesions while 3 were intraventricular. Intraparenchymal lesions were located in frontal lobe in 4 patients $(30.8 \%)$, temporal lobe in 2 patients, frontoparietal region in 2 patients, parietal lobe in one patient and thalamus in one patient. Intraventricular lesions were in the left occipital horn in 2 patients and right occipital horn in one patient. Based on maximum diameter, the lesions varied in size from 33 to $65 \mathrm{~mm}$ (mean $45.5 \mathrm{~mm}$ ).
The tubular retractor, which was used in this study, is a commercially available transparent plastic pediatric anoscope with an inner diameter of $18 \mathrm{~mm}$. (Sapimed, S.p.A. Alessandria, Italy). It has an inner obturator that is longer than the retractor itself (Figure $1 A-C$ ). The lenght of the tube is $54 \mathrm{~mm}$. To decrease the distance between the distal tips of the tube and the obturator, plastic stopper (plastic piston obtained from a $20 \mathrm{ml}$ plastic syringe) was placed at the end of the obturator (Figure 1C).

\section{Surgical Technique}

Preoperatively, an image-guided fine-cut Magnetic resonance imaging (MRI) scan with intravenous contrast was obtained to identify the appropriate site of the craniotomy and a safe cortical entry. After induction of anesthesia, the head was fixed with a Mayfield 3 pins head holder and the head position was adapted to the tumor location and then registered with the navigation system (StealthStation $®$; Medtronic, Inc., USA). Once the safe entry point and the best trajectory into the tumor were established, a small lazy S-shaped scalp incision was made large enough to allow for a craniotomy of approximately $3 \mathrm{~cm}$ in diameter (Figure 2). Following a small craniotomy, the dura was opened in a cruciate fashion. The corticotomy was made roughly equal to the diameter of the retractor tube. If possible, transsulcal approach was selected. After performing the corticotomy, the pediatric anoscope containing the obturator and the navigation probe inserted along with the lumen of the obturator was advanced slowly together into the brain along the desired trajectory with intermittent stereotactic feedback to ensure appropriate placement. The process of cannulation and the typical intraoperative view are demonstrated in Figures $3 \mathrm{~A}-\mathrm{C}$. The goal of cannulation was to arrive at approximately $1 / 3$ the depth of the tumor (if resistance was not encountered). However, the cannulation was typically targeted just passed the ependymal surface for ventricular lesions. Once the target point has been reached, 
the navigation probe and the introducer were removed together leaving the plastic tube in place. From this instance, an operative assistant was responsible for the manual control of the tubular retractor. A $30 \mathrm{~cm}$ long and $4 \mathrm{~mm}$ in diameter endoscope (Karl Storz, Inc., Tuttlingen, Germany) was then inserted through the working channel and it was held by the same assisting surgeon. Resection is performed using bimanual microsurgical techniques and various microsurgical instruments including an ultrasonic aspirator probe (Figure 3C). Firstly, a significant internal tumor debulking was performed and then as debulking proceeded, the direction of the retractor was adjusted multiple times to visualize the remnants and the margins of the lesion. After achieving the desired resection of the lesion, meticulous hemostasis was provided in the tumor bed. Because of the transparent nature of the retractor, the bleeding points in the surrounding brain parenchyma could be easily visualized and controlled while the tubular retractor was gradually withdrawn. The dura was then closed in watertight fashion, with or without a dural greft. The bone flap and skin were closed in a standard fashion.

Postoperative computed tomography (CT) scans were routinely obtained in all our patients on the first postoperative day, and subsequent scans were performed when needed. MRI scan with and without intravenous contrast was also obtained within 2 days after surgery to evaluate the extent of resection. By comparing preoperative MRI scans with early postoperative scans obtained within $48 \mathrm{~h}$ of surgery, gross-total resection was defined as no evidence of residual enhancement (Figure 4A-F), near-total resection was defined as removal of $95 \%$ or more of the lesions (Figure $5 \mathrm{~A}-\mathrm{F}$ ), and subtotal resection was defined as removal of less than $95 \%$
(7, 16). According to our clinical protocol MRI scan was performed regularly 3,6 , and 12 months after surgery in the first year, every 6 months for the next year, then annually.

\section{RESULTS}

With the use of this simple, inexpensive transparent pediatric anoscope as a tubular retractor under the navigation guidance, targeted lesions were successfully approached and the preoperative goals of surgery were met in all patients. Table I summarizes the histological diagnosis and extent of resection for 13 patients. This tubular retractor may accommodate a 4-mm rigid endoscope and two microsurgical instruments while permitting the use of standard microsurgical techniques through minimally invasive craniotomies. This surgical technique is performed by two surgeons skilled in endoscopic surgery, and minimizes the scalp incision associated with small craniotomy size. Because the tubular retractor is handheld throughout the surgery, the scope and tube angles could be adjusted multiple times in order to facilitate dynamic lesion visualization. This strategy allowed for bimanual manipulation of the tumor and facilitated removal of lesions much larger than diameter of the tube itself.

The histological diagnosis were as follows; 2 metastases, 5 glioblastomas, 3 meningiomas, 2 low-grade gliomas and one oligodendroglioma. With regards to extent of resection, gross total radiographic resection of the lesions was achieved in 7 of 13 patients $(53.8 \%)$, near-total resection was achieved in 3 of 13 patients $(23.1 \%)$. This means that effective resection of lesions could be achieved in 10 of 13 (76.9\%) cases using the endoscope and the simple tubular retractor, through small
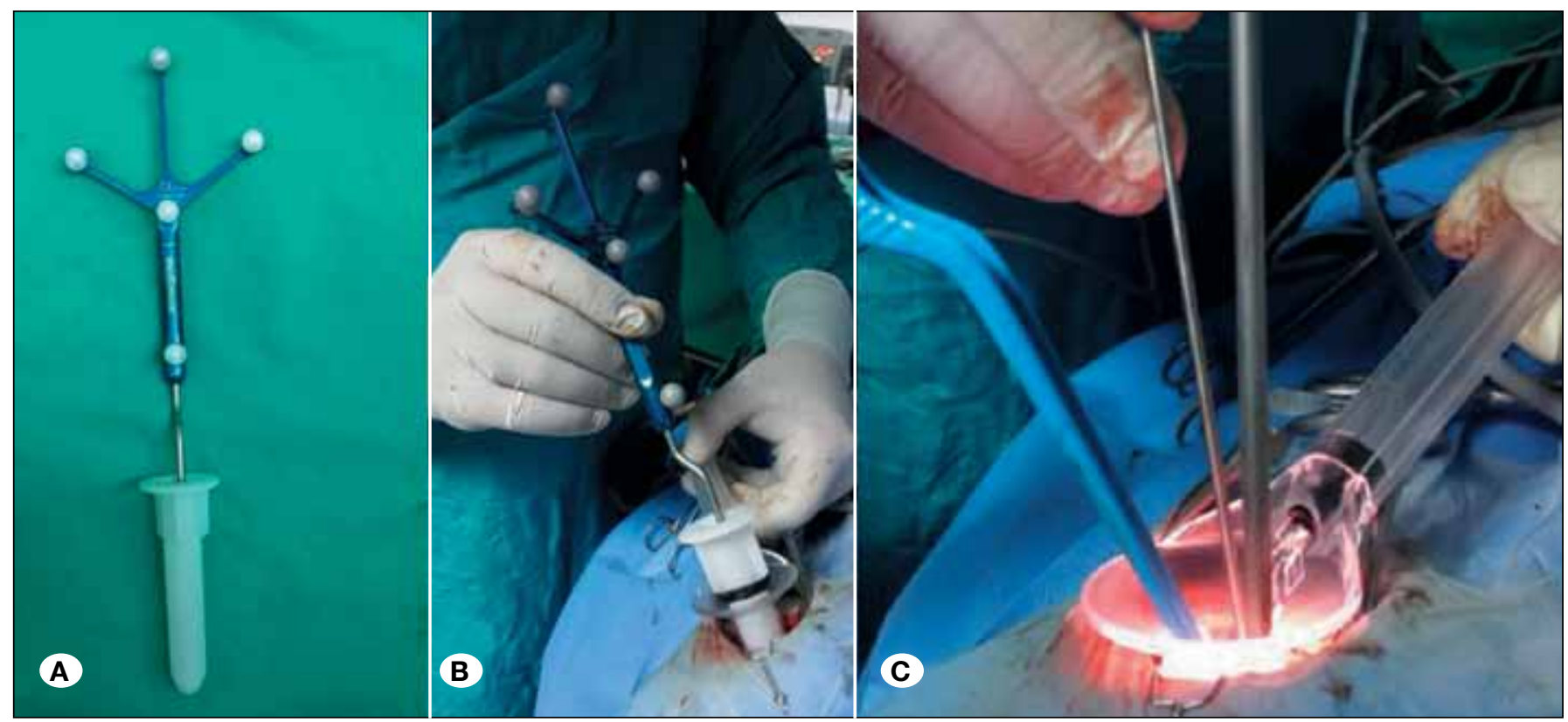

Figure 3: A) Photograph demonstrating the pediatric anoscope containing the obturator and the navigation probe inserted along with the lumen of the obturator. B) Intraoperative photograph showing insertion of the tubular retractor under image guidance until the target is reached. C) Intraoperative photograph showing the simultaneous use of bipolar forceps and suction through the tubular retractor during tumor excision. 


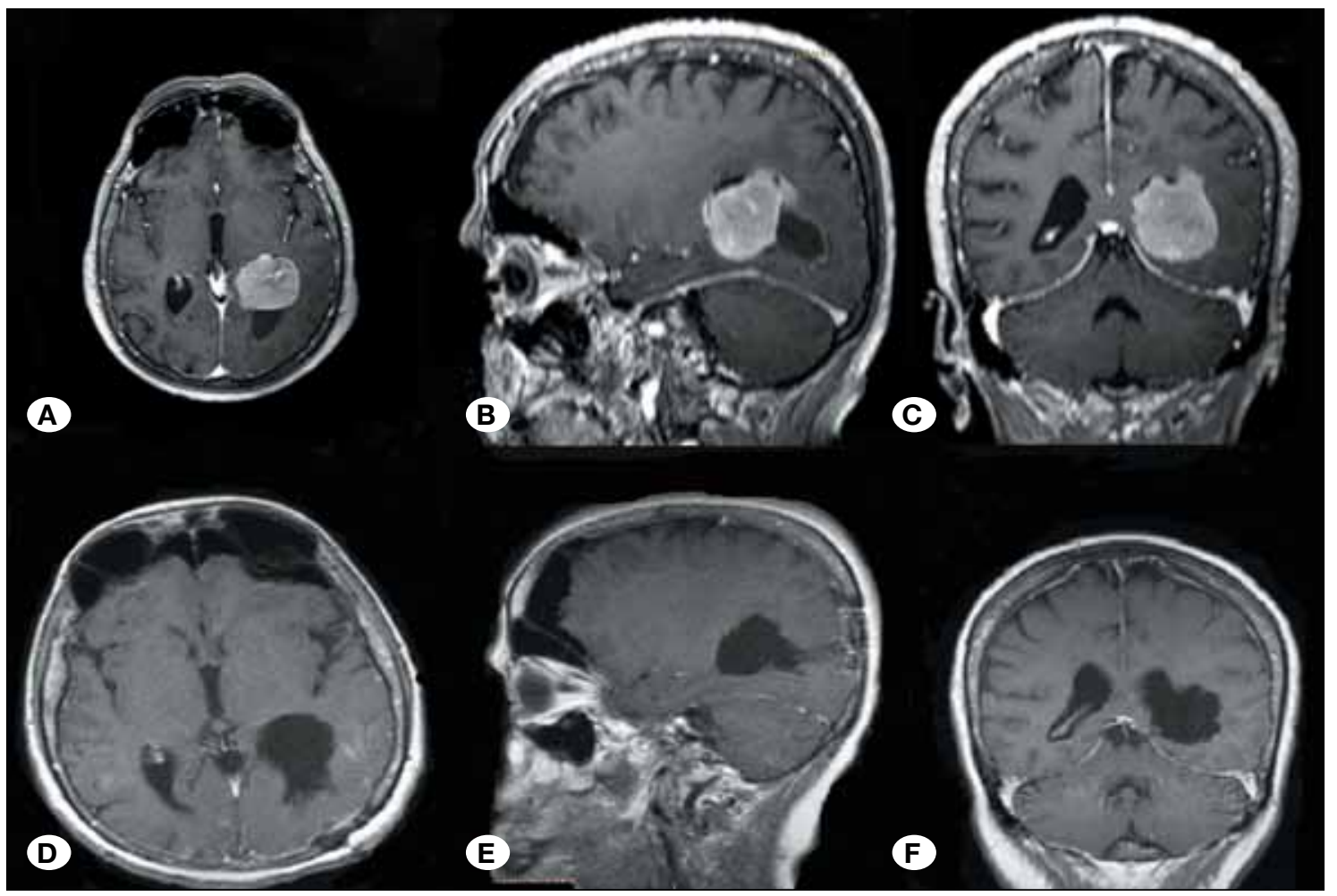

Figure 4: Preoperative T1-weighted axial (A), sagittal (B) and coronal (C) magnetic resonance imaging of the patient with left intraventricular mass lesion. The tumor was totally removed using endoscopic transcortical approach. The histological diagnosis was meningioma. Postoperative T1-weighted axial (D), sagittal (E) and coronal (F) magnetic resonance images confirm the total removal of tumor.

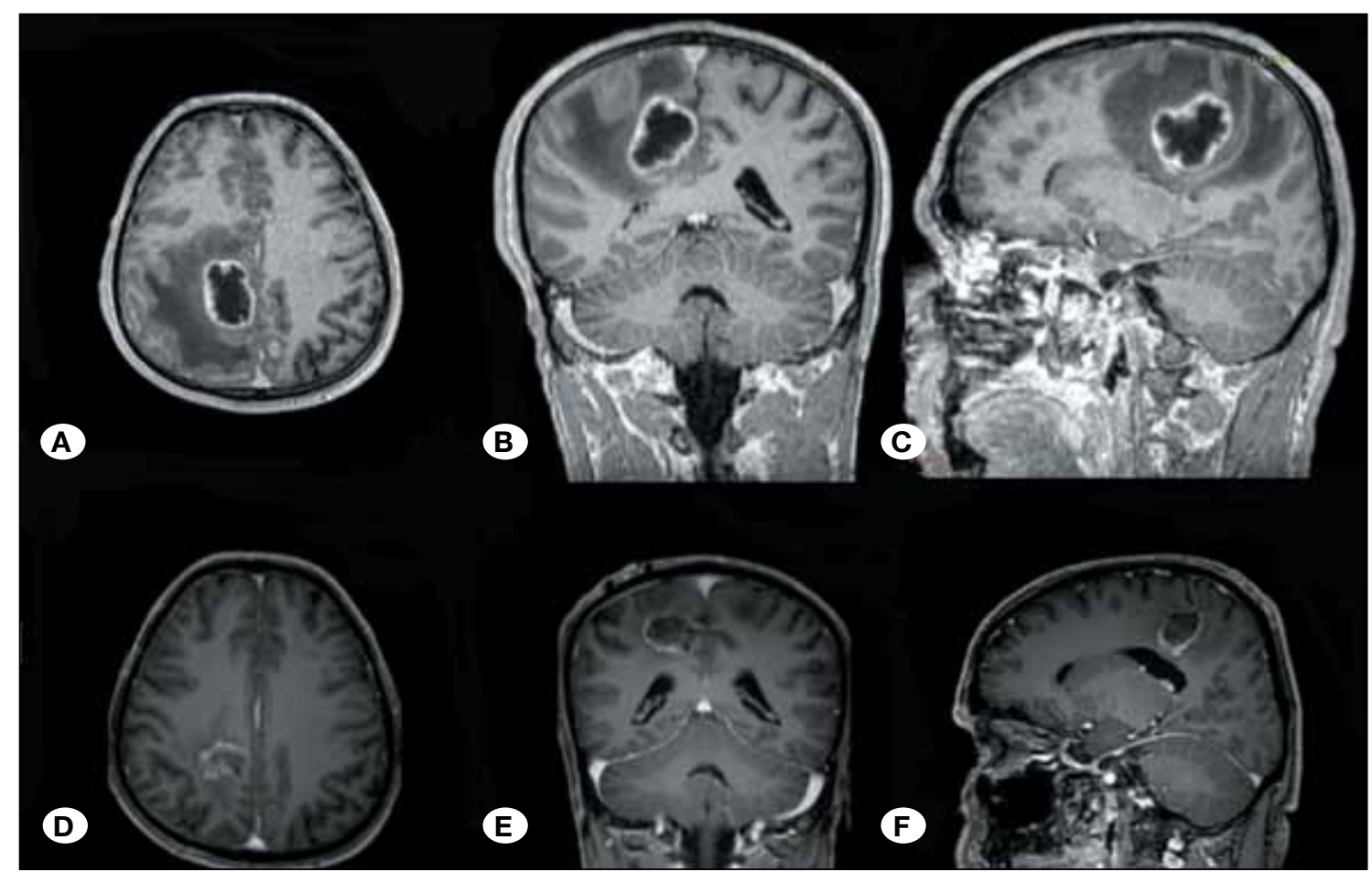

Figure 5: Preoperative T1-weighted axial (A), coronal (B) and sagittal (C) magnetic resonance imaging of the patient with right parietal intraparenchymal mass lesion. The tumor was removed using endoscopic transcortical approach. The histological diagnosis was metastatic carcinoma. Postoperative T1-weighted axial (D), coronal (E) and sagittal (F) magnetic resonance images confirm the neartotal removal of tumor. The peritumoral edema was also improved after surgery. 
size craniotomy window and small cortical incision. Subtotal removal was achieved in $3(23.1 \%)$ patients. For those patients, the decision was made to avoid total resection of the lesion due to involvement of eloquent areas. Duration of surgery ranged from 60 to 110 minutes, with an average 90 minutes.

There were no complications directly caused by the use of the endoscope or the tubular retractor. None of the patients demonstrated new neurological deficits or other postoperative complications such as hematomas in the surgical field, seizures, CSF leaks and also no hematomas were observed along the retractor tract on postoperative imaging.

Most patients are discharged home on postoperative day 2 or 3 . The average postoperative hospital stay was 2,7 days at which point the patient was discharged in stable neurological status. Follow-up intervals varied from 3 months to 24 months (median 5 months).

\section{DISCUSSION}

Deep-seated brain lesions such as intraventricular and intraparenchymal pathologies have traditionally been removed under direct microscopic visualization that utilize retraction of deep white matter to maintain a long passage traversing through surrounding white matter. However, the improper use of traditional flat retractors and a long-duration brain retraction can cause some complications such as seizures, focal neurologic deficits, vascular damage, cerebral swelling, and cognitive impairment $(14,29,30,33,37)$. The incidence of brain retraction injury varies from $5 \%$ to $10 \%(12,30,36,37)$.

With the use of such retractors, which do not exert equally distributed pressure, overly aggressive brain-retraction can cause significant damage to the surrounding brain tissue. Injury is often a result of direct pressure and local ischemia $(30,37)$. The stress created by retractor blades on the surrounding brain tissue, causes an increases of local cerebral tissue pressure and a decrease of regional cerebral blood flow $(33,36)$. Rosenorn et al. $(29,30)$ have documented a considerable reduction in regional cerebral blood flow with a retraction pressure of $25-30 \mathrm{mmHg}$, the threshold at which tissue infarction occurs.

In an effort to avoid these retraction-related complications, the concept of a tubular retraction system was introduced in the late 1980s $(18)$. Kelly et al. $(18,19)$ first described the use of a tubular system for stereotactic resection of intracranial tumors. A similar device was later introduced by Otsuki et al. (25), and then by Ross (31) and by Barlas and Karadereler (5) for use with a Leksell frame. In 2005, the neuroendoport system was originally applied by Harris et al. (11), to approach intraventricular tumors. This technique was a modification of the 'vinyl tube technique' previously described by Jho and Alfieri (13). In 2008, Greenfield et al. (10) have described the use of the METRX ${ }^{\circledR}$ tubular retractor system, (Medtronic Inc, Memphis, TN, USA) designed specifically for spinal surgery, for the microsurgical resection of ten deep intracranial lesions guided by a frameless navigation device. The ViewSite $\AA$ tubular brain retractor (Vycor, Inc.NY, USA) designed specifically for intracranial surgery is one of the another commercially available transparent tubular retractor system. The efficacy and safety of this tubular retractor system were documented by several study groups $(12,27,28)$.

Afterwards, many kinds of tubular retractors have been also developed for use with frameless stereotactic navigation systems $(10,14,15,24)$. Although the majority of these operations were performed under direct microscopic visualization, in the last years the minimally invasive techniques were increasingly dominated by endoscopy. The use of endoscopy in the management of various intraventricular, extra-axial and also skull base lesions is now a widely accepted therapeutic option (1, 6-9, 11, 13, 26, 35). Additionally, the advent of endoport or tubular retractor systems has also facilitated the biopsy or removal of deep-seated intraparenchymal lesions in an endoscopically controlled fashion. Although endoscopic visualization and removal of intraaxial brain tumors using stereotactic guidance was first described in 1980 (32), this area has continued to expand slowly. In 2008, Akai et al. (1), used a transparent tubular retractor to endoscopic resection of intrinsic brain tumors from 3 patients.

One year later, Kassam et al. (16) also reported their complication-free results after completely endoscopic resection of 21 deep-seated intra-axial lesions with the use of a nonfixed transparent conduit. Recently, several groups have reported their experience with this technique in the management of deeper intracranial lesions $(2,15,16,20-23,25,34)$. Review of the related literature has shown that tubular retractors seem to have some advantages over the conventional retractors $(8,10,30,33)$. They distribute pressure equally in order to minimize injury $(2,10,11,15,19,20,24)$. While the tubular sheath distributes the retraction forces evenly over the entire surface of the sheath, conventional spatula retractors localize pressure at the point of contact with brain parenchyma $(10,11$, $20,28)$. Therefore the low retracting pressure around a tubular retractor is not likely to be as disruptive as is the pressure in conventional flat-bladed retractors (24). In the study by Ogura et al. (24) the brain retraction pressure was $<10 \mathrm{mmHg}$ with the use of a tubular retractor. It has also been documented radiologically (based on T2 / FLAIR and diffusion restriction/ apparent diffusion coefficient signals, MRI based) that the tubular retractor systems place minimal stress on the surrounding parenchyma $(10,27,28)$ Greenfield at al. (10) have shown that postoperative edema, as judged by the FLAIR sequence on $\mathrm{MRI}$, was not worse than preoperative imaging in any patient.

In addition to these advantages of the tubular retractors, endoscopic surgery has also some advantages over conventional surgery performed under direct microscopic visualization. Endoscopic surgical views are wider (panoramic) and more detailed than microscopic views. It provides superior magnification and illumination which is helpful to differentiate between normal tissue and residual tumor $(1,14,15)$. Since microscopes provide an image along a linear corridor, the angle of surgical view can be adjusted only to a certain extent which may be lead to a limitation when visualizing deep structures along a long surgical corridor (4, 23, 34). Contrary 
to this, the endoscope can be used to work within the cavity, allowing visualization of structures around surgical corners and through small openings $(4,34,35)$. However, the use of the endoscope in conjunction with tubular retractors for the biopsy and resection of selected deep-seated lesions has not become widely practiced mainly due to some problems related to the technical aspect of tubular retractors. Most tubular retractors have their own limitations including the inability to use without frame-based or frameless stereotactic systems (not available in all neurosurgical centers), lack of transparency of the retractor walls because of their bulky and metallic or hard plastic nature, uncontrollable enlargement of the surgical corridor, the limited maneuverability, prolonged installation time, difficulties in their surgical manipulations, and fixation problems, their high-priced (5, 7-12, 15, 21-25, 27, 33).

In the endoscopic resection of deep-seated brain lesion, use of the tubular retractor combined with the neuronavigation system is essential $(1,11,16,22,24,31)$. Using the tubular retractor along with the navigation probe enables the surgeon to effectively target smaller and deeper lesions via minimally invasive corridors $(1,16,22)$ but this combination may be cumbersome for some of the tubular systems. The combination of the tubular retractor system with the intraoperative navigation system offers several surgical benefits, which include small scalp incision, mini-craniotomy with limited dural and brain exposure, less blood loss, short surgical time, minimal postoperative analgesic requirement, and reduced hospital length of stay $(1,2,7,9,10,16,22)$. Our simple and inexpensive tubular retractor, a modified pediatric anoscope was easily and successfully combined with frameless image guidance. All lesions were accessible surgically and all surgical goals could be achieved.

The other drawback of tubular retractors is the fixation problem. Many of the tubular retractor systems need to be secured (2, $16,17,24,30,34)$. During the operation, the working cannula is secured to the scalp to prevent its migration into the brain and also held in position using a conventional neurosurgical selfretractor system such as the Greenberg or Leyla bar systems. Moreover, some of them need their unique setbacks for their fixations $(2,10,11,15,20,23,27,28)$. When repositioning is necessary, the retractor has to be loosened and redirected, which is a time-consuming procedure. This kind of fixation also hampers maneuverability of the cannula. However, its well-known that endoscopic surgery proceeds as a dynamic process, with multiple adjustments of the port trajectory to facilitate resection $(16,23,33)$. Our tubular retractor is free and does not require any rigid holder. An operative assistant is needed to maintain the trajectory of the port and also steer the port. With the aid of this handle, the endoscopic view can be changed many times throughout the operation to facilitate dynamic resection, and this practice avoids time wastage in repositioning. The manual control allows dynamic mobilization of the working cannula with the endoscopic port in any plane during the procedure facilitates removal of lesions much larger than cannula itself. In this study, with the aid of dynamic mobilization of the cannula, tumors much larger than $3 \mathrm{~cm}$ in diameter could be resected safely. Additionally, tube's funnel- shaped entry acting as a shield is another advantage of this simple retractor. During the operation, this shield protected the brain surface and vessels against iatrogenic injuries caused by endoscope and instruments being passed in and out of the corridor.

Transparency is very important for a tubular retractor. Some of them are made of metal or plastic with opaque wallshindering visualization of surrounding tissue $(5,8,10,18,19$, 25). Additionally, metallic tubes can also conduct electricity. On the other hand, some of the transparent tubes are very soft and malleable. Thus, with the use of these soft retractors, maintenance of the surgical corridor can be challenging and also there may be some additional problems include difficulty in inserting into the brain and the need for additional retractors-fixation systems to hold the retractor in place $(5$, $10,13,15,24,25,28,33)$. Conversely, our retractor tube is lightweight and also provides transparent view of surrounding brain, hence early detection of contusions or bleeding in the surrounding brain is possible. Because of its plastic nature, electrical transmission is not possible, and at the same time it has enough sturdiness to maintain the surgical corridor. In our small series of adult patients, the use of a pediatric anoscope as a tubular retractor facilitated our surgical approach. Thirteen patients with intra-axial lesions larger than $3 \mathrm{~cm}$ in diameter were operated by this method with no new neurologic deficits. There were also no complications directly caused by the use of the endoscope and the tubular retractor. Patients' quick recovery, minimal postoperative discomfort, and short hospital stays have been observed. The results are comparable to what has been reported in the related literature $(2,11,15,16,20,34)$. This simple pediatric anoscope provides non fixed and easily tiltable retraction, and allows bimanual use of surgical instruments so that tumor can be easily resected using standard microsurgical techniques. Furthermore, unlike other commercially available systems which are expensive, our simple retractor is cheap, and available in most institutions or hospitals. The convertibility is an another advantage of this technique. The procedure can be easily converted to a standard microsurgical approach. One can switch to microscopic surgery and the rest of the operation can be performed under microscopic visualization, if needed. However, the procedure was not converted to a standard microsurgical approach in any of the patients in our series.

Finally, the results of this series should be interpreted with caution. The aim of this study was not to convince neurosurgeons that all intra-axial lesions can be treated with the endoscopic surgery. In the management of patients with deep-seated brain lesions, multiple approaches are available. We do not suggest that any of these resections required tubular retractors, only that this technology may be of use in select patients. It should be kept in mind that choosing the correct patient is essential for success and the endoportguided endoscopic surgery would not always be less invasive than the technique of a skilled neurosurgeon who uses the brain retractor effectively and safely. 


\section{- CONCLUSION}

The results in the present series suggest that endoscopic surgery using a transparent tubular retractor guided by a neuronavigation system facilitates the removal of deep-seated brain lesions. The pediatric anoscope used in our patients provides non fixed and easily tiltable retraction during the surgey. With the aid of dynamic mobilization of this simple retractor, tumors much larger than the tube can be effectively removed. This endoscopic approach may be a feasible alternative for brain tumor resections in selected cases, especially deep-seated large lesions

\section{- REFERENCES}

1. Akai T, Shiraga S, Sasagawa Y, Okamoto K, Tachibana O, Lizuka $\mathrm{H}$ : Intra-parenchymal tumor biopsy using neuroendoscopy with navigation. Minim Invasive Neurosurg 51: 83-86, 2008

2. Almenawer SA, Crevier L, Naresh M, Kassam A, Reddy K: Minimal access to deep intracranial lesions using a serial dilatation technique. Neurosurg Rev 36(2):321-329; discussion 329-330,2013

3. Apuzzo MLJ, Heifetz MD, Weiss MH, Kurze T: Neurosurgical endoscopy using the side-viewing telescope: Technical note. J Neurosurg 46: 398-400,1977

4. Badie B, Brooks N, Souweidane MM: Endoscopic and minimally invasive microsurgical approaches for treating brain tumor patients. J Neuro-Oncology 69: 209-219, 2004

5. Barlas $O$, Karadereler S: Stereotactically guided microsurgical removal of colloid cysts. Acta Neurochir (Wien) 146(11):11191204, 2004

6. Cappabianca P, Cavallo LM, Colao A, Del Basso de Caro M, Esposito F, Cirillo S, Lombardi G, de Divitiis E: Endoscopic endonasal transsphenoidal approach: Outcome analysis of 100 consecutive procedures. Minim Invasive Neurosurg 45: 193-200, 2002

7. Engh JA, Lunsford LD, Amin DV, Ochalski PG, FernandezMiranda J, Prevedello DM, Kassam AB: Stereotactically guided endoscopic port surgery for intraventricular tumor and colloid cyst resection. Neurosurgery 67(3):198-205, 2010

8. Fahim DK, Relyea K, Nayar VV, Fox BD, Whitehead WE, Curry DJ, Luersen TG, Jea A: Transtubular microendoscopic approach for resection of a choroidal arteriovenous malformation J Neurosurg Pediatr 3(2):101-104,2009

9. Fries G, Perneczky A: Endoscope-assisted brain surgery: Part 2-analysis of 380 procedures. Neurosurgery 42: 226-231,1998

10. Greenfield JP, Cobb WS, Tsouris AJ, Schwartz TH: Stereotactic minimally invasive tubular retractor system for deep brain lesions. Neurosurgery 63(4):334-340,2008

11. Harris AE, Hadjipanayis CG, Lunsford LD, Lunsford AK, Kassam AB: Microsurgical removal of intraventricular lesions using endoscopic visualization and stereotactic guidance. Neurosurgery 56(1):125-132, 2005

12. Herrera SR, Shin JH, Chan M, Kouloumberis P, Goellner E, Slavin KV: Use of transparent plastic tubular retractor in surgery for deep brain lesions: A case series. Surg Technol Int $19: 47-50,2010$
13. Jho HD, Alfieri A: Endoscopic removal of third ventricular tumors: A technical note. Minim Invasive Neurosurg 45(2):114119,2002

14. Jo KI, Chung SB, Jo KW, Kong DS, Seol HJ, Shin HJ: Microsurgical resection of deep-seated lesions using transparent tubular retractor: Pediatric case series. Childs Nerv Syst 27(11):1989-1994,2011

15. Jo KW, Shin HJ, Nam DH, Lee JI, Park K, Kim JH, Kong DS: Efficacy of endoport-guided endoscopic resection for deepseated brain lesions. Neurosurg Rev 34(4):457-463,2011

16. Kassam AB, Engh JA, Mintz AH, Prevedello DM: Completely endoscopic resection of intraparenchymal brain tumors. J Neurosurg 110:116-123,2009

17. Kassam AB, Snyderman $\mathrm{CH}$, Mintz AH, Gardner P, Carrau RL: Expanded endonasal approach: The rostro caudal axis. Part 1. Crista gali to the sella turcica. Neurosurg Focus 19(1):E3,20 05

18. Kelly PJ, Goeres SJ, Kall BA: The stereotaxic retractor in computer assisted stereotaxic microsurgery. Technical note. J Neurosurg 69: 301-306,1988

19. Kelly PJ, Kall BA, Goerss SJ: Computer-interactive stereotactic resection of deep-seated and centrally located intraaxial brain lesions. Appl Neurophysiol 50(1-6):107-113,1987

20. McLaughlin N, Prevedello DM, Engh J, Kelly DF, Kassam AB: Endoneurosurgical resection of intraventricular and intraparenchymal lesions using the port technique. World Neurosurg 79(2 Suppl):S18.e1-8, 2013

21. Miranda JCF, Engh JA, Pathak SK, Madhok R, Boada FE, Schneider W, Kassam AB: High-definition fiber tracking guidance for intraparenchymal endoscopic port surgery. J Neurosurg 113:990-999,2010

22. Nakano T, Ohkuma H, Asano K, Ogasawa Y: Endoscopic treatment for deep-seated or multiple intraparenchymal tumors: Technical note. Minim Invasive Neurosurg 52:4952,2009

23. Ochalski PG, Engh JA: Endoscopic port surgery for intraparenchymal brain tumors. In: Hayat MA (ed), Tumors of the Central Nervous System, Vol: 3. Springer: Science+Business Media BV, 2011:60-267

24. Ogura K, Tachibana E, Aoshima C, Sumitomo M: New microsurgical technique for intraparenchymal lesions of the brain: Transcylinder approach. Acta Neurochir (Wien) 148:779785; discussion 785, 2006

25. Otsuki T, Jokura H, Yoshimoto T: Stereotactic guiding tube for open-system endoscopy a new approach for the stereotactic endoscopic resection of intra-axial brain tumors. Neurosurgery 27: 326-330,1990

26. Perneczky A, Fries G: Endoscope-assisted brain surgery: Part 1-evolution, basic concept, and current technique. Neurosurgery 42: 219-224,1998

27. Raza SM, Recinos PF, Avendano J, Adams H, Jallo GI, Quinones-Hinojosa JA: Minimally invasive trans-portal resection of deep intracranial lesions. Minim Invas Neurosurg 54:5-11,2011

28. Recinos PF, Raza SM, Jallo GI, Recinos VR: Use of a minimally invasive tubular retraction system for deep-seated tumors in pediatric patients. J Neurosurg Pediatrics 7(5):516-521,2011 
29. Rosenorn J, Diemer NH: Reduction of cerebral blood flow during brain retraction pressure in the rat. J Neurosurg 56: 826-829,1982

30. Rosenorn J, Diemer NH: The risk of cerebral damage during graded brain retraction pressure in the rat. $\mathrm{J}$ Neurosurg 63:608-611,1985

31. Ross DA: A simple stereotactic retractor for use with the Leksell stereotactic system. Neurosurgery 32(3):475-476,1993

32. Shelden $\mathrm{CH}, \mathrm{McCann} \mathrm{G}$, Jacques S, Lutes HR, Frazier RE, Katz $\mathrm{R}$, Kuki R: Development of a computerized microstereotaxic method for localization and removal of minute CNS lesions under direct 3-D vision. Technical report. J Neurosurg 52: 2127,1980
33. Singh L, Agrawal N: Cylindrical channel retractor for intraventricular tumour surgery- a simple and inexpensive device. Acta Neurochir (Wien) 151(11):1493-1497,2009

34. Teo C, Nakaji P: Application of endoscopy to the resection of intra-axial tumors. Oper Tech Neurosurg 8:179-185,2005

35. Teo C, Nakaji P: Neuro-oncologic applications of endoscopy. Neurosurg Clin N Am 15:89-103,2004

36. Yokoh A, Sugita K, Kobayashi S: Intermittent versus continuous brain retraction: An experimental study. J Neurosurg 58:918923,1983

37. Zhong J, Dujovny M, Perlin AR, Perez-Arjona E, Park HK, Diaz FG: Brain retraction injury. Neurol Res 25(8):831-838, 2003 